\title{
Phosphorylated mammalian target of rapamycin expression is associated with the response to chemoradiotherapy in patients with esophageal squamous cell carcinoma
}

\author{
Shau-Hsuan Li, MD, ${ }^{\text {a,e }}$ Eng-Yen Huang, MD,,${ }^{\text {be }}$ Hung-I Lu, MD, ${ }^{\text {c,e }}$ Wan-Ting Huang, MD, ${ }^{\text {d }}$ \\ Chueh-Chuan Yen, MD, ${ }^{\mathrm{f}}$ Wen-Chien Huang, MD, ${ }^{\mathrm{g}}$ and Chang-Han Chen, $\mathrm{PhD}^{\mathrm{e}, \mathrm{h}}$
}

\begin{abstract}
Objective: The mammalian target of rapamycin signaling pathway has been implicated in therapeutic resistance in several types of cancer. However, the significance of mammalian target of rapamycin activation in chemoradiotherapy sensitivity and its effect on the prognosis of esophageal squamous cell carcinoma treated with chemoradiotherapy remain unknown. However, this pathway is of particular interest because an effective inhibitor is available.
\end{abstract}

\begin{abstract}
Methods: By using immunohistochemistry, phosphorylated mammalian target of rapamycin expression was examined in 77 patients with esophageal squamous cell carcinoma treated with preoperative chemoradiotherapy followed by surgery between 1999 and 2009, and correlated with treatment outcome. With the use of CE81T/ VGH and TE2 cell lines, cells were treated with chemotherapy, temsirolimus (mammalian target of rapamycin inhibitor), or a combination of chemotherapy and temsirolimus, and investigated by 3-(4.5-dimethylthiazol2-yl)-2,5-diphenyltetrazolium bromide assay.
\end{abstract}

Results: Pathologic complete response rates were $42 \%$ and $16 \%$ in patients with negative and positive phosphorylated mammalian target of rapamycin expression, respectively $(P=.01)$. The 3 -year overall survivals were $57 \%$ and $30 \%$ in patients with negative and positive phosphorylated mammalian target of rapamycin expression, respectively $(P=.005)$. Positive phosphorylated mammalian target of rapamycin expression was independently associated with inferior overall and disease-free survival. In patients who did not achieve pathologic complete response, postchemoradiotherapy esophagectomy specimens showed significantly higher phosphorylated mammalian target of rapamycin expression than pretreatment biopsy specimens. In cell lines, concomitant administration of temsirolimus enhanced the effect of chemotherapy.

Conclusions: Phosphorylated mammalian target of rapamycin expression is independently associated with the response to chemoradiotherapy and prognosis of patients with esophageal squamous cell carcinoma treated with preoperative chemoradiotherapy. Mammalian target of rapamycin inhibition can sensitize esophageal cancer cells to chemotherapy. Our results suggest the potential for mammalian target of rapamycin as a therapeutic target for patients with esophageal squamous cell carcinoma who receive multimodality treatment. (J Thorac Cardiovasc Surg 2012;144:1352-9)

Supplemental material is available online.

The outcome of patients with advanced esophageal squamous cell carcinoma (ESCC) treated with surgery alone is unsatisfactory. ${ }^{1,2}$ In an attempt to improve survival, a multimodality approach using combined chemoradiotherapy with and

\footnotetext{
From the Department of Hematology-Oncology, ${ }^{\mathrm{a}}$ Department of Radiation Oncology, ${ }^{\mathrm{b}}$ Department of Thoracic and Cardiovascular Surgery, ${ }^{\mathrm{c}}$ and Department of Pathology, ${ }^{\mathrm{d}}$ Kaohsiung Chang Gung Memorial Hospital and Chang Gung University College of Medicine, Kaohsiung, Taiwan, ROC; Kaohsiung Chang Gung Esophageal Cancer Group, e Cancer Center, Kaohsiung Chang Gung Memorial Hospital and Chang Gung University College of Medicine, Kaohsiung, Taiwan, ROC; Division of Hematology and Oncology, ${ }^{\mathrm{f}}$ Department of Medicine, Taipei Veterans General Hospital and National Yang-Ming University School of Medicine, Taipei, Taiwan, ROC; Division of Thoracic Surgery, ${ }^{\mathrm{g}}$ Department of Surgery, Mackay Memorial Hospital, Mackay Medicine, Nursing, and Management College, Taipei, Taiwan, ROC; and Center for Translational Research in Biomedical Sciences, ${ }^{\text {h }}$ Kaohsiung Chang Gung Memorial Hospital and Chang Gung University College of Medicine, Kaohsiung, Taiwan, ROC.
}

without surgery has been advocated for patients. ${ }^{3,4}$ However, the value of the surgery after chemoradiotherapy remains unclear. Recent phase III trials ${ }^{3,4}$ showed that surgery may not be necessary for those patients who respond well to chemoradiotherapy. After preoperative chemoradiotherapy, pathologic complete response is found in only $20 \%$ to $40 \%$ of patients ${ }^{3-6}$ and is associated with improved survival. ${ }^{7}$ However, there is still a large portion of

\footnotetext{
Funding: This work was supported in part by grants from the National Science Council, Taiwan (NSC 100-2314-B-182A-044-MY3) and Chang Gung Memorial Hospital (CMRPG890631).

Disclosures: Authors have nothing to disclose with regard to commercial support.

Received for publication March 10, 2012; revisions received May 18, 2012; accepted for publication June 18, 2012; available ahead of print July 27, 2012.

Address for reprints: Chang-Han Chen, $\mathrm{PhD}$, Kaohsiung Chang Gung Esophageal Cancer Group, Cancer Center, and Center for Translational Research in Biomedical Sciences, Kaohsiung Chang Gung Memorial Hospital and Chang Gung University College of Medicine, 123 Ta-Pei Rd, Niaosung Hsiang, Kaohsiung Hsien, Taiwan, ROC (E-mail: chench7@gmail.com).

$0022-5223 / \$ 36.00$

Copyright (C) 2012 by The American Association for Thoracic Surgery http://dx.doi.org/10.1016/j.jtcvs.2012.06.049
} 


$$
\begin{aligned}
& \text { Abbreviations and Acronyms } \\
& \text { AJCC = American Joint Committee on Cancer } \\
& \text { CDI = combination drug index } \\
& \text { CI = confidence interval } \\
& \text { CT = computed tomography } \\
& \text { DFS = disease-free survival } \\
& \text { DMEM = Dulbecco's Modified Eagle Medium } \\
& \text { ESCC = esophageal squamous cell carcinoma } \\
& \text { EUS }=\text { endoscopic ultrasound } \\
& \text { 5-FU }=5 \text {-fluorouracil } \\
& \text { mTOR = mammalian target of rapamycin } \\
& \mathrm{OR}=\text { odds ratio } \\
& \text { OS = overall survival } \\
& \text { p-mTOR }=\text { phosphorylated mammalian target of } \\
& \text { rapamycin }
\end{aligned}
$$

patients who do not respond well to chemoradiotherapy. In addition, there are substantial differences in the response to chemoradiotherapy and survival between patients with the same clinical stage. Therefore, there is an urgent need to identify patients who are likely to respond to chemoradiotherapy to spare them the potential perioperative morbidity and mortality. A reliable marker that can precisely predict the tumor response to chemoradiotherapy in patients with ESCC is important, and this marker may serve as a therapeutic target in the future.

The mammalian target of rapamycin (mTOR), also known as "FK506 binding protein 12-rapamycin associated protein 1," is a serine/threonine protein kinase that plays a key role in regulating protein synthesis, ribosomal protein translation, and cap-dependent translation. ${ }^{8}$ Deregulation in mTOR signaling is frequently associated with tumorigenesis, angiogenesis, tumor growth, and metastasis. ${ }^{8}$ In response to extracellular stimuli, mTOR is activated by phosphorylation of Ser2448 through the phosphatidylinositol 3-kinase/AKT signaling pathway. ${ }^{8}$ Activation of mTOR stimulates the phosphorylation of 2 key downstream proteins that regulate protein translation: eukaryotic initiation factor $4 \mathrm{E}$ binding protein-1 and protein S6 kinase 1. Through the activation of eukaryotic initiation factor $4 \mathrm{E}$ and protein S6 kinase 1, mTORC complex 1 promotes the translation of messenger RNAs that often encode proteins necessary for cell cycle progression, cell growth, and metabolism. A number of studies suggest that activation of the mTOR signaling pathway plays an important role in multiple anticancer drug-resistance mechanisms in several types of cancer. ${ }^{9}$ In particular, a recent development of the mTOR inhibitor temsirolimus has generated considerable excitement in both the clinical and the basic cancer research communities, because it exhibits potent activity against a wide panel of cancers. ${ }^{10}$ Therefore, further elucidation of the mTOR activation in human cancers is critical for anticancer therapy targeting the mTOR signaling pathway. However, the significance of mTOR activation in chemoradiotherapy sensitivity and its effect on the prognosis of patients with ESCC treated with chemoradiotherapy remain unclear.

Thus, we evaluated the mTOR activity with immunohistochemistry and investigated the prognostic role of mTOR activation in 77 patients with ESCC who were treated with preoperative chemoradiotherapy followed by surgery. In addition, we also use ESCC cell lines to determine whether the mTOR inhibitor can enhance the effect of chemotherapy.

\section{MATERIALS AND METHODS \\ Patient Population}

Patients with ESCC who were treated with chemoradiotherapy at Kaohsiung Chang Gung Memorial Hospital between January 1999 and December 2009 were retrospectively reviewed. This study was approved by the institutional review board of Chang Gung Memorial Hospital. All patients selected for this study were required to undergo esophagectomy after chemoradiotherapy to allow pathologic correlation.

During this period, 109 patients with ESCC who were treated with preoperative chemoradiotherapy followed by surgery were identified. Among these 109 patients, 25 (23\%) achieved pathologic complete response. Pretreatment specimens of biopsy were available in 77 patients. Patients were evaluated by a multidisciplinary team, including a thoracic surgeon, medical oncologist, radiation oncologist, radiologist, and gastroenterologist. Pretreatment staging evaluation included physical examination, endoscope, contrast-enhanced computed tomography (CT) scans from the neck to upper abdomen, and endoscopic ultrasound (EUS). All 77 patients had CT scans. EUS was performed in 61 patients. In patients who did not receive EUS, $\mathrm{T}$ stage was determined by CT scans only. The clinical staging was determined according to the American Joint Committee on Cancer (AJCC) staging system (AJCC Cancer Staging Manual, 7 th Edition). ${ }^{11}$

\section{Treatment Plan}

Patients were treated with 2 cycles of cisplatin and 5-fluorouracil (5-FU)-based chemotherapy and radiotherapy (36 Gy in 20 fractions) concurrently. The interval between treatment cycles was 3 to 4 weeks. Radiotherapy of 1.8 Gy per day was delivered 5 days per week for 4 weeks. Three-dimensional conformal radiotherapy via a 4-field technique was used in most cases. Within 3 to 4 weeks after the end of irradiation, a CT scan was performed to assess the treatment response. The multidisciplinary team reviewed the clinical information to determine whether the lesions were resectable. If yes, surgery was advised approximately 4 to 6 weeks after the end of preoperative chemoradiotherapy. Patients undergoing surgery had a radical esophagectomy with cervical esophagogastric anastomosis (McKeown procedure) or an Ivor Lewis esophagectomy with intrathoracic anastomosis, reconstruction of the digestive tract with gastric tube, and pylorus drainage procedures. Two-field lymph node dissection was performed in all patients. Pathologic complete response was defined as the complete disappearance of all viable cancer cells in all surgical specimens, including the primary esophageal tumor and lymph nodes.

\section{Patient Follow-up}

After the operation, patients were scheduled for follow-ups at 3-month intervals in years 1 and 2, 6-month intervals in years 3 to 5, and annually 
thereafter. Overall survival (OS) was calculated from the date of diagnosis to death as a result of all causes. Disease-free survival (DFS) was computed from the time of surgery to the recurrence or death from any cause without evidence of recurrence.

\section{Immunohistochemistry}

Immunohistochemistry staining was performed using an immunoperoxidase technique. Staining was performed on slides $(4 \mu \mathrm{m})$ of formalinfixed, paraffin-embedded tissue sections with primary antibodies against phosphorylated mTOR (p-mTOR) (Ser2448, Clone 49F9, 1:50, Cell Signaling Technology, Inc, Danvers, Mass). Briefly, after deparaffinization and rehydration, the retrieval of the antigen was performed by treating the slides in $10 \mathrm{mmol} / \mathrm{L}$ citrate buffer $(\mathrm{pH} 6.0)$ in a hot water bath $\left(95^{\circ} \mathrm{C}\right)$ for 20 minutes. Endogenous peroxidase activity was blocked for 15 minutes in $0.3 \%$ hydrogen peroxide. After blocking with $1 \%$ goat serum for 1 hour at room temperature, the sections were incubated with primary antibodies for at least 18 hours at $4{ }^{\circ} \mathrm{C}$ overnight. Immunodetection was performed using the LSAB2 kit (Dako, Carpinteria, Calif) followed by 3-3'-diaminobenzidine for color development and hematoxylin for counterstaining. Incubation without the primary antibody and normal esophageal squamous epithelium ${ }^{12}$ were used as a negative control. A normal gastric gland ${ }^{12}$ was used as a positive control. The staining assessment was independently carried out by 2 pathologists (S.L.W. and W.T.H) without any information about clinicopathologic features or prognosis. The fraction of tumor cells with cytoplasmic p-mTOR expression $(0 \%$ $100 \%$ ) was recorded, and the average value of 2 pathologists was calculated in each patient. Whenever the 2 pathologists disagreed (eg, difference $>10 \%$ or discordant scores), the slides were reevaluated and discussed, and a consensus was reached by 2 pathologists. Then p-mTOR expression level was scored by using the 3-tier system: negative expression (score 0), less than $10 \%$; low expression (score 1), $10 \%$ or more, but less than $50 \%$; and high expression (score 2), $50 \%$ or more tumor cells with staining. ${ }^{13,14}$ When scores were classified into 2 groups for statistical analysis, "low" and "high" were combined as "positive.", 12-14

\section{Cell Culture and 3-(4.5-Dimethylthiazol-2-yl)-2,5- \\ Diphenyltetrazolium Bromide Assay}

Human esophageal cancer cells (CE81T/VGH), a cell line derived from a well-differentiated squamous cell carcinoma of the esophagus, were provided by Dr C-C Yen (Taipei Veterans General Hospital, Taipei, Taiwan) and cultured in Dulbecco's Modified Eagle Medium (DMEM) supplemented with $10 \%$ fetal calf serum, $2 \mathrm{mmol} / \mathrm{L}$ glutamine, $100 \mathrm{U} /$ $\mathrm{mL}$ penicillin, and $100 \mathrm{ig} / \mathrm{mL}$ streptomycin. Human esophageal cancer cells (TE2), a cell line derived from a poorly differentiated squamous cell carcinoma of the esophagus, were provided by Dr W-C Huang (Mackay Memorial Hospital, Taipei, Taiwan) and cultured in DMEM supplemented with $10 \%$ fetal calf serum, $2 \mathrm{mmol} / \mathrm{L}$ glutamine, $100 \mathrm{U} /$ $\mathrm{mL}$ penicillin, and $100 \mathrm{ig} / \mathrm{mL}$ streptomycin. The rapamycin analogue CCI-779 (temsirolimus) was provided by Wyeth-Ayerst (King of Prussia, Pa).

To test the effects of cell proliferation of chemotherapy alone and in combination with temsirolimus on cell proliferation, cells were plated into 96-well, flat-bottomed plates at $3 \times 10^{3}$ cells per $100 \mu \mathrm{L}$ per well in DMEM containing $10 \%$ fetal bovine serum. After overnight incubation, triplicate wells were treated with chemotherapy alone, temsirolimus alone, or chemotherapy in combination with different concentrations of temsirolimus for 24 hours. The relative percentages of metabolically active cells compared with untreated controls were then determined on the basis of mitochondrial conversion of 3-(4.5-dimethylthiazol-2-yl)-2,5diphenyltetrazolium bromide to formazine. In brief, after incubation, $10 \mu \mathrm{L}$ of 3-(4.5-dimethylthiazol-2-yl)-2,5-diphenyltetrazolium bromide (Sigma, St Louis, Mo) solution $(5 \mathrm{mg} / \mathrm{mL}$ ) was added to each well for 3 hours, and then the medium was replaced with $150 \mu \mathrm{L}$ of dimethyl sulfoxide per well. Results were assessed in a 96-well format plate reader by measuring the absorbance at a wavelength of $540 \mathrm{~nm}$ using a Titertek Multiscan (Thermo, Finland).

\section{Statistical Analysis}

For patient data, statistical analysis was performed using the Statistical Package for the Social Sciences 17 software package (SPSS Inc, Chicago, Ill). The chi-square and Fisher exact tests were used to compare data between the 2 groups. Changes in p-mTOR expression during chemoradiotherapy were analyzed by the McNemar-Bowker test. Multivariate analysis of pathologic complete response was performed by logistic regression, and variables with $P$ values less than .1 identified at the univariate level were entered into the model. For survival analysis, the Kaplan-Meier method was used for univariate analysis, and the difference between survival curves was tested by a log-rank test. In a stepwise forward fashion, parameters with $P$ values less than .1 at the univariate level were entered into the Cox regression model to analyze their relative prognostic importance. For all analyses, 2-sided tests of significance were used.

For ESCC cell line experiments, $t$ test was used for the statistical analysis. Data are presented as means \pm standard deviation. Each experiment was carried out independently at least twice, with 3 repeats each. The combination drug index (CDI) was used to evaluate whether the combination of drug A and drug B was synergistic or not, which was calculated according to the following formula ${ }^{15}$ : CDI $=$ survival $\%($ drug $\mathrm{A}+$ drug $\mathrm{B}) /$ survival $\%($ drug A $) \times$ survival $\%($ drug $B)$. The value of CDI less than 1 meant synergistic drug interaction.

\section{RESULTS \\ Patient Characteristics}

A total of 77 patients were collected in the study with a median age of 52 years (range, 37-77 years). Among them, 75 were men and 2 were women. The pretreatment analyses of the clinical tumor stage revealed $\mathrm{T} 2$ in 4 patients $(5 \%)$, T3 in 39 patients $(51 \%)$, and $\mathrm{T} 4$ in 34 patients $(44 \%)$. Additional pretreatment analyses according to the AJCC staging system demonstrated clinical stage II tumors for 16 patients $(21 \%)$ and clinical stage III for 61 patients $(79 \%)$. Further analyses of histologic grades showed a grade 1 lesion in 12 patients, grade 2 in 44 patients, and grade 3 in 21 patients. Primary tumor location was found to be upper in 13 patients $(17 \%)$, middle in 38 patients $(49 \%)$, and lower in 26 patients $(34 \%)$.

Among these 77 patients, 21 achieved pathologic complete response after preoperative chemoradiotherapy. The 3 -year OS and DFS for these 77 patients were $42 \%$ and $38 \%$, respectively. The median periods of follow-up were 73.2 months (range, 28.6-120 months) for the 24 survivors and 22.6 months (range, 3.8-120 months) for all 77 patients.

\section{Correlation Between Clinicopathologic Parameters and Expression of Phosphorylated Mammalian Target of Rapamycin}

p-mTOR immunoreactivity was absent in normal squamous cell epithelium (Figure 1, $A$ ) but was present in normal gastric glands (Figure 1, B). Among the 77 patients 

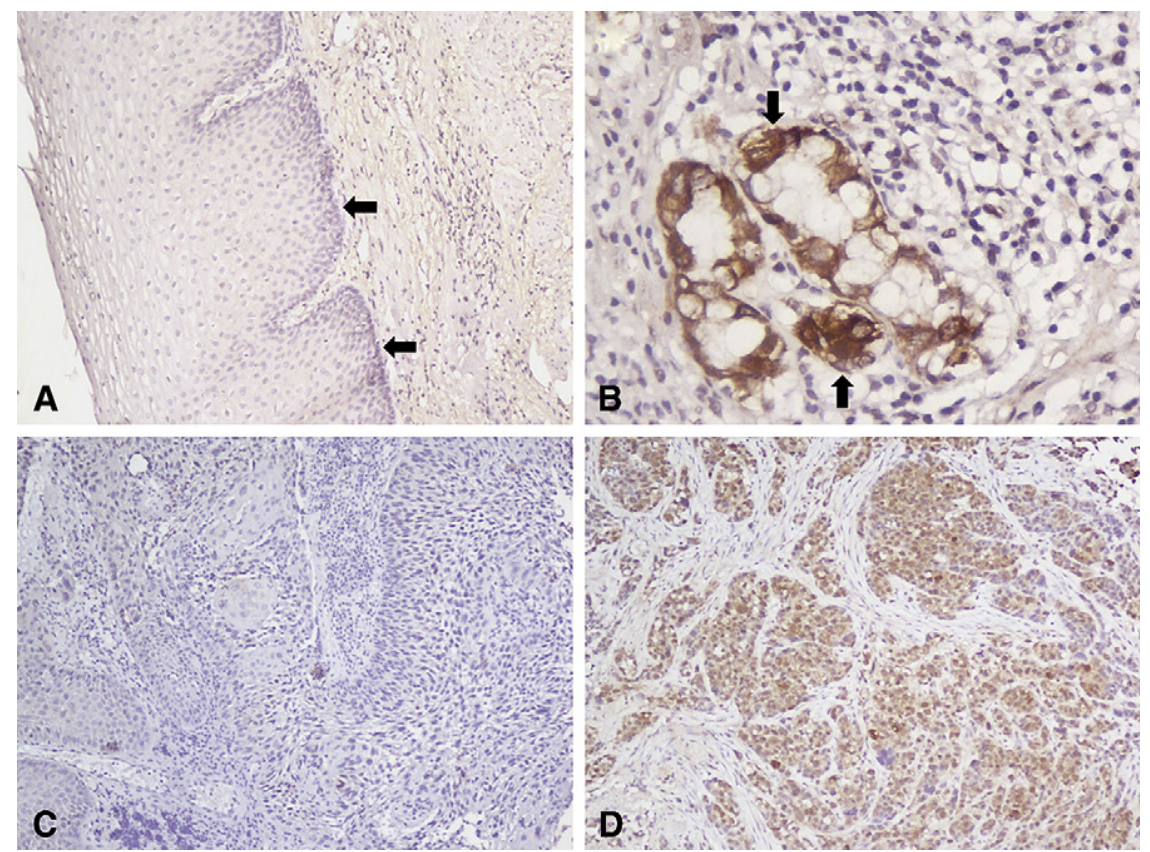

FIGURE 1. Immunohistochemical staining of p-mTOR. A, p-mTOR immunoreactivity was absent in normal squamous cell epithelium (black arrows). Original magnification $\times 200$. B, p-mTOR immunoreactivity was present in normal gastric glands (black arrows). Original magnification $\times 400$. C, Representative example of negative p-mTOR expression in ESCC. Original magnification $\times 100$. D, Representative example of positive p-mTOR expression in ESCC. Original magnification $\times 100$.

collected, 33 (43\%) showed "negative" for p-mTOR expression and $44(57 \%)$ showed "positive" for p-mTOR (Figure 1, $C$ and $D$ ). p-mTOR expression was not associated with any clinicopathologic parameters, including age, gender, primary tumor location, histologic grade, keratinizing, AJCC staging, $\mathrm{T}$ staging, and $\mathrm{N}$ staging (data not shown).

\section{Correlation Between Clinicopathologic Parameters and Pathologic Complete Response}

The relationship between the clinicopathologic parameters and the response to chemoradiotherapy is summarized in Table 1. Negative p-mTOR expression $(P=.01)$ was significantly associated with pathologic complete response after chemoradiotherapy. The pathologic complete response rate was $42 \%$ in patients with negative p-mTOR expression compared with $16 \%$ in patients with positive p-mTOR expression. The logistic model showed that negative p-mTOR expression $(P=.012$; odds ratio [OR], 3.891; $95 \%$ confidence interval [CI], 1.346-11.236) independently correlated with pathologic complete response after chemoradiotherapy.

\section{Survival Analyses}

Correlations of clinicopathologic parameters and p-mTOR expression with OS and DFS are summarized in Table 2. Univariate analyses demonstrated that positive p-mTOR expression (3-year OS rate, $30 \%$ vs $57 \%, P=.005$; 3 -year DFS rate,
$25 \%$ vs $54 \%, P=.015$; Figure $2, A$ and $B$ ), AJCC stage III (3-year OS rate, $34 \%$ vs $68 \%, P=.015$; 3 -year DFS rate, $30 \%$ vs $68 \%, P=.007$ ), T4 disease (3-year OS rate, $27 \%$ vs $54 \%, P=.01$; 3-year DFS rate, $24 \%$ vs $49 \%$, $P=.007)$, and positive regional lymph nodes (3-year OS rate, $33 \%$ vs $61 \%, P=.019 ; 3$-year DFS rate, $28 \%$ vs $61 \%, P=.005)$ were associated with the inferior OS and DFS. In multivariate comparison, positive p-mTOR expression (OS, $P=.001$; OR, 2.814; 95\% CI, 1.553-5.097; DFS, $P=.003$; OR, $2.438 ; 95 \%$ CI, 1.368-4.347), T4 disease (OS, $P=.005$; OR, 2.256; 95\% CI, 1.276-3.990; DFS, $P=.005$; OR, $2.227 ; 95 \%$ CI, 1.271-3.902), and positive regional lymph nodes $(\mathrm{OS}, P=.015$; OR, $2.329,95 \% \mathrm{CI}$, 1.180-4.598; DFS, $P=.003$; OR, 2.749; 95\% CI, 1.399 5.402) represented independent adverse prognosticators for OS and DFS. The 3-year OS and DFS were $30 \%$ and $25 \%$, respectively, in patients with positive p-mTOR expression, and $57 \%$ and $54 \%$, respectively, in patients with negative p-mTOR expression.

\section{Change of the Phosphorylated Mammalian Target of Rapamycin Expression After Chemoradiotherapy}

Among the 77 patients collected, 56 did not achieve pathologic complete response after chemoradiotherapy. Immunohistochemical p-mTOR expression scores were compared between the biopsy samples obtained before chemoradiotherapy and the esophagectomy specimens after chemoradiotherapy. Esophagectomy specimens after 
TABLE 1. Associations between pathologic complete response and clinicopathologic parameters

\begin{tabular}{|c|c|c|c|}
\hline \multirow[b]{2}{*}{ Parameters } & \multicolumn{3}{|c|}{ Pathologic complete response } \\
\hline & Absent & Present & $P$ value \\
\hline \multicolumn{4}{|l|}{ Age } \\
\hline$<52$ y & 25 & 11 & .54 \\
\hline$\geq 52$ y & 31 & 10 & \\
\hline \multicolumn{4}{|l|}{ AJCC stage } \\
\hline II & 9 & 7 & .12 \\
\hline III & 47 & 14 & \\
\hline \multicolumn{4}{|l|}{ T stage } \\
\hline $\mathrm{T} 2+3$ & 28 & 15 & .092 \\
\hline $\mathrm{T} 4$ & 28 & 6 & \\
\hline \multicolumn{4}{|l|}{$\mathrm{N}$ stage } \\
\hline No & 14 & 9 & .127 \\
\hline $\mathrm{N} 1+2+3$ & 42 & 12 & \\
\hline \multicolumn{4}{|l|}{ Tumor grade } \\
\hline $1+2$ & 38 & 18 & .12 \\
\hline 3 & 18 & 3 & \\
\hline \multicolumn{4}{|c|}{ Primary tumor location } \\
\hline Upper/middle & 38 & 13 & .62 \\
\hline Lower & 18 & 8 & \\
\hline \multicolumn{4}{|l|}{ Keratinizing } \\
\hline Absent & 11 & 4 & 1.00 \\
\hline Present & 45 & 17 & \\
\hline \multicolumn{4}{|l|}{ p-mTOR } \\
\hline$<10 \%$ & 19 & 14 & $.01^{*}$ \\
\hline$\geq 10 \%$ & 37 & 7 & \\
\hline
\end{tabular}

AJCC, American Joint Committee on Cancer; $p$-mTOR, phosphorylated mammalian target of rapamycin. *Statistically significant. Chi-square or Fisher exact test was used for statistical analysis.

chemoradiotherapy showed significantly $(P=.004$, Figure $2, C$ ) higher percentages of high p-mTOR scores than the biopsy samples before chemoradiotherapy.

\section{Mammalian Target of Rapamycin Inhibitor \\ Potentiates Growth Inhibition by Chemotherapy in \\ Esophageal Squamous Cell Carcinoma Cell Lines}

Our clinical data showed that p-mTOR expression is significantly associated with the response to chemoradiotherapy. Therefore, we tested whether the mTOR inhibitor temsirolimus can potentiate growth inhibition by chemotherapy in ESCC cell lines, CE81T/VGH and TE2. We compared the ability of the mTOR inhibitor temsirolimus to exhibit cooperative effects with cisplatin or 5-FU using doses of chemotherapy that resulted in approximately $15 \%$ to $30 \%$ growth inhibition when given alone. Temsirolimus alone displayed a growth-inhibitory effect in a dose-dependent manner (Figure E1). In addition, a combination of chemotherapy and temsirolimus showed significantly better growth inhibition than chemotherapy or temsirolimus alone (Figure 3). Synergistic effects were also observed, especially in TE2 ESCC cell lines.
TABLE 2. Results of univariate log-rank analysis of prognostic factors for overall and disease-free survivals in 77 patients with esophageal squamous cell carcinoma receiving preoperative chemoradiotherapy followed by surgery

\begin{tabular}{|c|c|c|c|c|c|}
\hline \multirow[b]{2}{*}{ Factors } & \multirow[b]{2}{*}{$\begin{array}{c}\text { No. of } \\
\text { patients }\end{array}$} & \multicolumn{2}{|c|}{ OS } & \multicolumn{2}{|c|}{ DFS } \\
\hline & & $\begin{array}{c}3-\mathbf{y} \\
\text { OS }(\%)\end{array}$ & $\begin{array}{c}P \\
\text { value }\end{array}$ & $\begin{array}{c}3-\mathbf{y} \\
\text { DFS (\%) }\end{array}$ & $\begin{array}{c}P \\
\text { value }\end{array}$ \\
\hline \multicolumn{6}{|l|}{ Age } \\
\hline$<52 \mathrm{y}$ & 36 & 44 & .51 & 39 & .66 \\
\hline$\geq 52 \mathrm{y}$ & 41 & 39 & & 37 & \\
\hline \multicolumn{6}{|l|}{ p-mTOR } \\
\hline$<10 \%$ & 33 & 57 & $.005^{*}$ & 54 & $.015^{*}$ \\
\hline$\geq 10 \%$ & 44 & 30 & & 25 & \\
\hline \multicolumn{6}{|l|}{ AJCC stage } \\
\hline II & 16 & 68 & $.015^{*}$ & 68 & $.007 *$ \\
\hline III & 61 & 34 & & 30 & \\
\hline \multicolumn{6}{|l|}{$\mathrm{T}$ classification } \\
\hline $\mathrm{T} 2+3$ & 43 & 54 & $.01 *$ & 49 & $.007 *$ \\
\hline $\mathrm{T} 4$ & 34 & 27 & & 24 & \\
\hline \multicolumn{6}{|l|}{$\mathrm{N}$ status } \\
\hline NO & 23 & 61 & $.019 *$ & 61 & $.005^{*}$ \\
\hline $\mathrm{N} 1+2+3$ & 54 & 33 & & 28 & \\
\hline \multicolumn{6}{|l|}{ Tumor grade } \\
\hline 1 & 12 & 58 & .35 & 58 & .34 \\
\hline $2+3$ & 65 & 38 & & 34 & \\
\hline \multicolumn{6}{|c|}{ Primary tumor location } \\
\hline Upper/middle & 51 & 39 & .48 & 35 & .49 \\
\hline Lower & 26 & 46 & & 42 & \\
\hline \multicolumn{6}{|l|}{ Keratinizing } \\
\hline Absent & 15 & 33 & .44 & 27 & .47 \\
\hline Present & 62 & 44 & & 40 & \\
\hline \multicolumn{6}{|l|}{ Pathologic CR } \\
\hline Absent & 56 & 30 & $.009 *$ & 25 & $.007 *$ \\
\hline Present & 21 & 71 & & 71 & \\
\hline
\end{tabular}

$O S$, Overall survival; $D F S$, disease-free survival; $p$ - $m T O R$, phosphorylated mammalian target of rapamycin; $A J C C$, American Joint Committee on Cancer; $C R$, complete response. *Statistically significant.

\section{DISCUSSION}

Previous studies have revealed that mTOR activation is associated with poor prognosis in numerous human cancers. ${ }^{16,17}$ However, the significance of mTOR activation in chemoradiotherapy sensitivity and its effect on the prognosis of patients with ESCC treated with chemoradiotherapy remain unknown. Thus, we focused on the patients with ESCC who were treated with preoperative chemoradiotherapy and undertook the present study to determine the significance of mTOR activation in chemoradiotherapy sensitivity and patients' survival.

In the present study, a significant inverse correlation between expression of p-mTOR and the response to chemoradiotherapy was found. A total of $14(42 \%)$ of 33 patients with negative p-mTOR expression achieved pathologic complete response after preoperative chemoradiotherapy, but only $7(16 \%)$ of 44 patients with positive p-mTOR expression achieved pathologic complete 


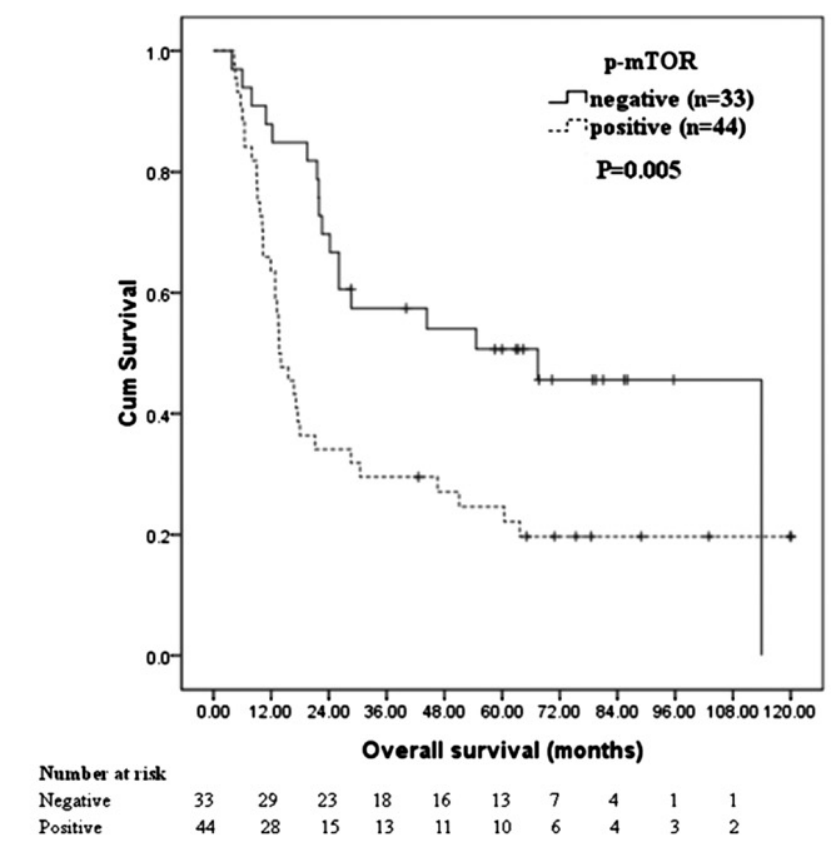

A

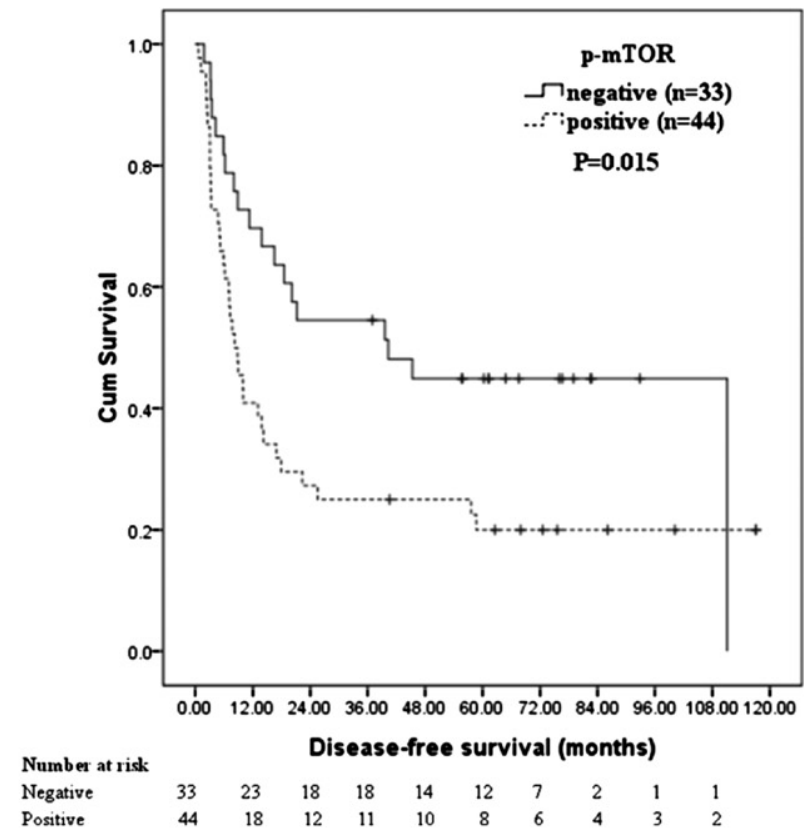

B

Changes in p-mTOR expression during CRT in 56
squamous cell carcinoma patients who received
preoperative CRT
\begin{tabular}{ccccccc}
\multicolumn{5}{c}{ After CRT } \\
\cline { 2 - 7 } p-mTOR score & 0 & 1 & 2 & Total \\
\hline \multirow{3}{*}{ Before CRT } & 1 & 3 & 10 & 6 & 19 \\
& 2 & 1 & 2 & 10 & 13 \\
& Total & 8 & 20 & 28 & 56 \\
C CRT, chemoradiotherapy: ${ }^{*}$ McNemar-Bowker Test
\end{tabular}
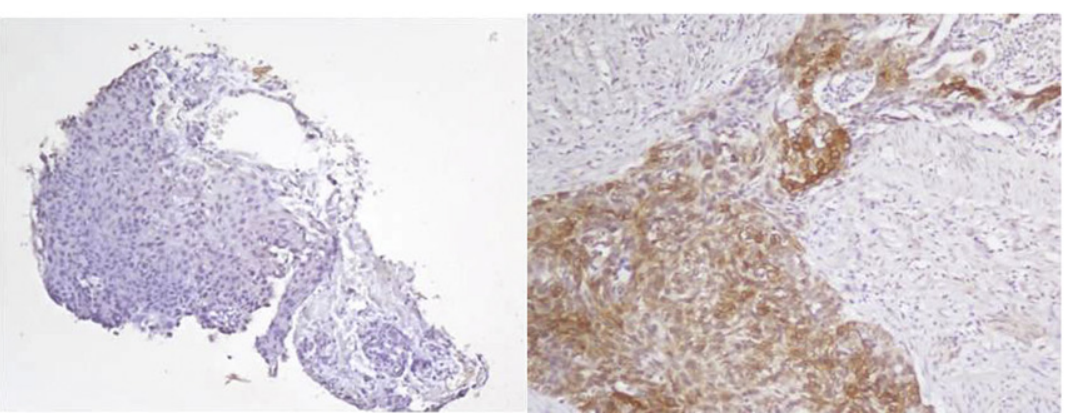

FIGURE 2. A, OS according to p-mTOR status. B, DFS according to p-mTOR status. C, Percentage of high p-mTOR scores was significantly higher in the esophagectomy specimens after chemoradiotherapy compared with biopsy specimens before chemoradiotherapy $(P=.004$, left $)$. Representative paired samples of a biopsy specimen (middle, -mTOR score $=0$, original magnification $\times 40$ ) and an esophagectomy specimen $($ right, $\mathrm{p}$-mTOR score $=2$, original magnification $\times 100$ ). $p$-mTOR, Phosphorylated mammalian target of rapamycin; $C R T$, chemoradiotherapy.

response after preoperative chemoradiotherapy. ESCC is usually diagnosed at an advanced stage. The use of preoperative chemoradiotherapy followed by surgery or definitive chemoradiotherapy has been increasing in recent years for patients with advanced ESCC. After chemoradiotherapy, no viable tumors are observed in $20 \%$ to $40 \%$ of resected esophageal tumor specimens. The therapeutic long-term benefit to achieve a pathologic complete response is suggested with multiple retrospective series reporting 5-year survivals of $40 \%$ to $60 \%$ in those groups of patients compared with those with specimens containing residual disease. ${ }^{7}$ On the other hand, perioperative morbidity and mortality may be increased by preoperative chemoradiotherapy. ${ }^{18}$ The ability to identify patients who cannot respond well to chemoradiotherapy may allow more appropriate selection of multimodality treatment options.
In our study, a significantly higher p-mTOR expression was found in the specimens obtained after the postchemoradiotherapy esophagectomy than the specimens collected before treatment. Previous studies report that mTOR activation after anticancer therapy plays an important role in protecting cells from undergoing apoptosis. ${ }^{9,19}$ Beuvink and colleagues ${ }^{20}$ recently reported that the inhibition of mTOR activation sensitized tumor cells to cisplatin-induced apoptosis through p53-induced p21 expression. Furthermore, the activation of mTOR after chemoradiotherapy in ESCC may also be involved in the stimulation of glycolysis. Warburg ${ }^{21}$ observed that cancer cells prefer glycolytic pathways with lactic acid fermentation in the cytosol to aerobic respiratory activity with mitochondrial oxidation of pyruvate for energy generation. This metabolic switch renders cancer cells less dependent on an oxygen supply and may allow them to survive in a more 

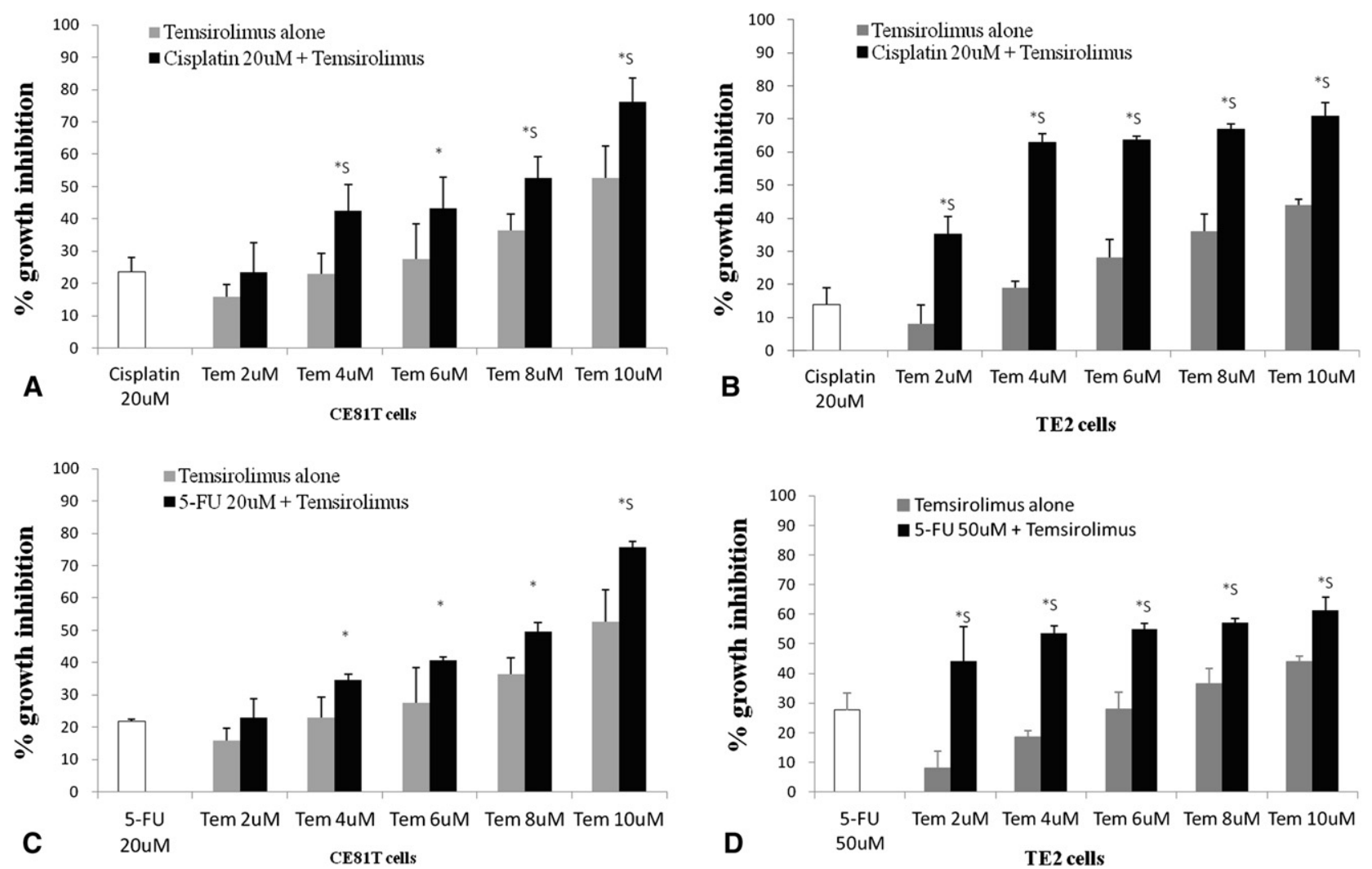

FIGURE 3. A and B, Temsirolimus inhibits cell growth in combination with cisplatin in CE81T and TE2 ESCC cell lines. CE81T and TE2 ESCC cell lines were treated with cisplatin alone, temsirolimus alone, or a combination of cisplatin and temsirolimus. Cisplatin was given at a single concentration. C and D, Temsirolimus inhibits cell growth in combination with 5-FU. CE81T and TE2 ESCC cell lines were treated with 5-FU alone, temsirolimus alone, or a combination of 5-FU and temsirolimus. 5-FU was given at a single concentration. *Significant difference in growth inhibition after combination treatment compared with chemotherapy or temsirolimus alone. Columns, mean; bars, standard deviation. 5-FU, 5-Fluorouracil; S, synergistic effect; Tem, temsirolimus.

hypoxic environment, such as an environment after chemotherapy or radiotherapy. This phenomenon may contribute to the resistance to chemotherapy and radiotherapy. Recent studies demonstrated that mTOR activation is critical for the metabolic switch to aerobic glycolysis of cancer cells. ${ }^{22}$

The mTOR inhibitor has been approved for clinical use in the systemic treatment of advanced renal cell carcinoma. ${ }^{9,10}$ Several preclinical studies have also suggested that the mTOR inhibitor can enhance the efficacy of a variety of chemotherapeutic agents in breast, ${ }^{23}$ ovarian, ${ }^{24}$ and colon ${ }^{25}$ cancers. However, little is known about the combination effect of the mTOR inhibitor and cytotoxic agents in ESCC. Hou and colleagues ${ }^{26}$ reported that the mTOR inhibitor rapamycin significantly inhibits the growth of ESCC when combined with cisplatin. In the present study, we found that the mTOR inhibitor temsirolimus enhances the effect of cisplatin and 5-FU, which are common chemotherapeutic agents used in ESCC. These results indicate that the mTOR inhibitor can enhance the potentiation of conventional chemotherapy and serve as a new therapeutic tool for patients with advanced ESCC.

\section{Study Limitations}

Our study has important limitations. Most of the patients in this study did not have fluorine 18 fluorodeoxyglucose positron emission tomography scans during pretreatment staging or in restaging after neoadjuvant chemoradiotherapy because 18 fluorodeoxyglucose-positron emission tomography scans are not routinely supported by Taiwan's health insurance. Furthermore, our observations were also limited by relatively short follow-up periods and small patient numbers.

\section{CONCLUSIONS}

Our study showed that p-mTOR expression is independently associated with the response to chemoradiotherapy and prognosis of patients with ESCC who were treated with preoperative chemoradiotherapy. Furthermore, inhibition of mTOR can sensitize esophageal cancer cells to chemotherapy. Therefore, mTOR may be a target for therapeutic intervention in patients with ESCC who receive multimodality treatment. 
The authors thank the Center for Translational Research in Biomedical Sciences, Kaohsiung Chang Gung Memorial Hospital (CLRPG871342) for providing the instruments for this study, and Sheng-Lan Wang (a pathologist from Union Pathology Center, Kaohsiung, Taiwan, ROC) for assessment of immunohistochemical staining.

\section{References}

1. Siewert JR, Stein HJ, Feith M, Bruecher BL, Bartels H, Fink U. Histologic tumor type is an independent prognostic parameter in esophageal cancer: lessons from more than 1,000 consecutive resections at a single center in the Western world. Ann Surg. 2001;234:360-9.

2. Lee PC, Mirza FM, Port JL, Stiles BM, Paul S, Christos P, et al. Predictors of recurrence and disease-free survival in patients with completely resected esophageal carcinoma. J Thorac Cardiovasc Surg. 2011;141:1196-206.

3. Stahl M, Stuschke M, Lehmann N, Meyer HJ, Walz MK, Seeber S, et al. Chemoradiation with and without surgery in patients with locally advanced squamous cell carcinoma of the esophagus. J Clin Oncol. 2005;23:2310-7.

4. Bedenne L, Michel P, Bouche O, Milan C, Mariette C, Conroy T, et al. Chemoradiation followed by surgery compared with chemoradiation alone in squamous cancer of the esophagus: FFCD 9102. J Clin Oncol. 2007;25:1160-8.

5. Meredith KL, Weber JM, Turaga KK, Siegel EM, McLoughlin J, Hoffe S, et al. Pathologic response after neoadjuvant therapy is the major determinant of survival in patients with esophageal cancer. Ann Surg Oncol. 2010;17:1159-67.

6. Meguid RA, Hooker CM, Taylor JT, Kleinberg LR, Cattaneo SM 2nd, Sussman MS, et al. Recurrence after neoadjuvant chemoradiation and surgery for esophageal cancer: does the pattern of recurrence differ for patients with complete response and those with partial or no response? J Thorac Cardiovasc Surg. 2009; 138:1309-17

7. Berger AC, Farma J, Scott WJ, Freedman G, Weiner L, Cheng JD, et al. Complete response to neoadjuvant chemoradiotherapy in esophageal carcinoma is associated with significantly improved survival. J Clin Oncol. 2005;23:4330-7.

8. Sabatini DM. mTOR and cancer: insights into a complex relationship. Nat Rev Cancer. 2006;6:729-34.

9. Jiang BH, Liu LZ. Role of mTOR in anticancer drug resistance: perspectives for improved drug treatment. Drug Resist Updat. 2008;11:63-76.

10. Edge SB, Byrd DR, Compton CC, Fritz AG, Greene FL, Trotti A, eds. Esophagus and esophagogastric junction. In: AJCC Cancer Satging Manual. 7th ed. New York: Springer; 2010:103-11.

11. Rini BI. Temsirolimus, an inhibitor of mammalian target of rapamycin. Clin Cancer Res. 2008; 14:1286-90.

12. Hirashima K, Baba Y, Watanabe M, Karashima R, Sato N, Imamura Y, et al. Phosphorylated mTOR expression is associated with poor prognosis for patients with esophageal squamous cell carcinoma. Ann Surg Oncol. 2010;17: 2486-93.

13. Dobashi Y, Suzuki S, Matsubara H, Kimura M, Endo S, Ooi A. Critical and diverse involvement of Akt/mammalian target of rapamycin signaling in human lung carcinomas. Cancer. 2009;115:107-18.

14. Dobashi Y, Suzuki S, Sato E, Hamada Y, Yanagawa T, Ooi A. EGFR-dependen and independent activation of Akt/mTOR cascade in bone and soft tissue tumors. Mod Pathol. 2009;22:1328-40.

15. Xu F, Zhen YS. (-)-Epigallocatechin-3-gallate enhances anti-tumor effect of cytosine arabinoside on HL-60 cells. Acta Pharmacol Sin. 2003;24:163-8.

16. Noh WC, Kim YH, Kim MS, Koh JS, Kim HA, Moon NM, et al. Activation of the mTOR signaling pathway in breast cancer and its correlation with the clinicopathologic variables. Breast Cancer Res Treat. 2008;110:477-83.

17. Moore SM, Rintoul RC, Walker TR, Chilvers ER, Haslett C, Sethi T. The presence of a constitutively active phosphoinositide 3-kinase in small cell lung cancer cells mediates anchorage-independent proliferation via a protein kinase B and p70s6k-dependent pathway. Cancer Res. 1998;58:5239-47.

18. Reynolds JV, Ravi N, Hollywood D, Kennedy MJ, Rowley S, Ryan A, et al. Neoadjuvant chemoradiation may increase the risk of respiratory complications and sepsis after transthoracic esophagectomy. J Thorac Cardiovasc Surg. 2006;132: 549-55.

19. Lefranc F, Facchini V, Kiss R. Proautophagic drugs: a novel means to combat apoptosis-resistant cancers, with a special emphasis on glioblastomas. Oncologist. 2007;12:1395-403.

20. Beuvink I, Boulay A, Fumagalli S, Zilbermann F, Ruetz S, O'Reilly T, et al. The mTOR inhibitor RAD001 sensitizes tumor cells to DNA-damaged induced apoptosis through inhibition of p21 translation. Cell. 2005;120:747-59.

21. Warburg O. On the origin of cancer cells. Science. 1956;123:309-14.

22. Sun Q, Chen X, Ma J, Peng H, Wang F, Zha X, et al. Mammalian target of rapamycin up-regulation of pyruvate kinase isoenzyme type M2 is critical for aerobic glycolysis and tumor growth. Proc Natl Acad Sci U S A. 2011;108: 4129-34.

23. Mondesire WH, Jian W, Zhang H, Ensor J, Hung MC, Mills GB, et al. Targeting mammalian target of rapamycin synergistically enhances chemotherapy-induced cytotoxicity in breast cancer cells. Clin Cancer Res. 2004;10:7031-42.

24. Itamochi H, Oishi T, Shimada M, Sato S, Uegaki K, Naniwa J, et al. Inhibiting the mTOR pathway synergistically enhances cytotoxicity in ovarian cancer cells induced by etoposide through upregulation of c-Jun. Clin Cancer Res. 2011;17: 4742-50.

25. Wagner M, Roh V, Strehlen M, Laemmle A, Stroka D, Egger B, et al. Effective treatment of advanced colorectal cancer by rapamycin and 5-FU/oxaliplatin monitored by TIMP-1. J Gastrointest Surg. 2009;13:1781-90.

26. Hou G, Zhang Q, Wang L, Liu M, Wang J, Xue L. mTOR inhibitor rapamycin alone or combined with cisplatin inhibits growth of esophageal squamous cell carcinoma in nude mice. Cancer Lett. 2010;290:248-54. 

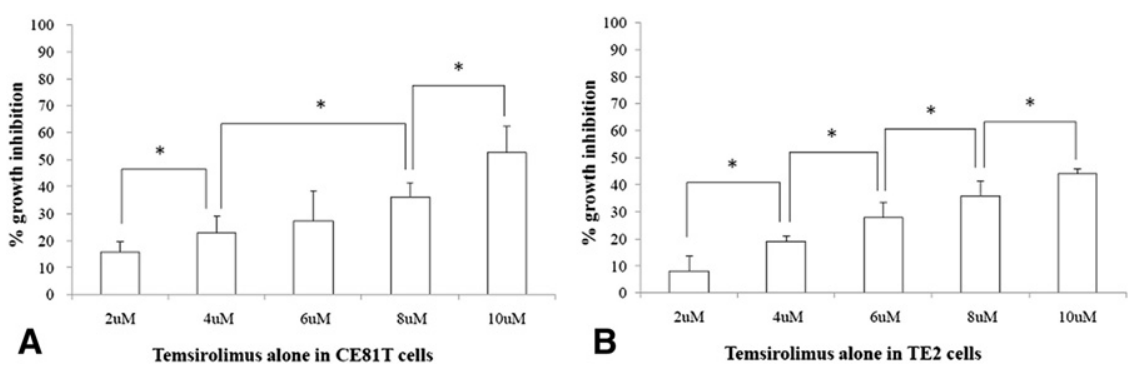

FIGURE E1. Temsirolimus alone displayed a growth-inhibitory effect in a dose-dependent manner in 2 ESCC cell lines: CE81T (A) and TE2 (B). *Significant difference in growth inhibition. Columns, mean; bars, standard deviation. 